\title{
Avolition in a patient with hypogonadism
}

\author{
Willem Verhoeven ${ }^{\star, \star *}$ \\ Siegfried Tuinier ${ }^{\star}$ \\ Jos Egger ${ }^{*, * * *}$ \\ Femke van Erp ${ }^{\star \star \star * \star}$ \\ Joep Tuerlings ${ }^{\star}$
}

* Vincent van Gogh Institute for Psychiatry, Department of Clinical Research, Venray

** Erasmus University Medical Centre, Department of Psychiatry, Rotterdam

*** Behavioural Science

Institute/Department of Psychology, Radboud University, Nijmegen

**** Department of Human Genetics, Radboud University Nijmegen, Medical Centre, Nijmegen

THE NETHERLANDS

\footnotetext{
ABSTRACT - Background and Objectives: Testosterone deficiency has been implicated in the etiology of depression although there is an ongoing debate on the nature of this association. There is a paucity of data about the psychological impact of hypogonadism in genetic disorders associated with testosterone deficiency.

Methods: A 57-year-old male is described who was referred for treatment resistant depression. His history showed infertility and lowered testosterone. A selective literature review is given to clarify the patient's clinical condition.

Results: Cytogenetic analysis demonstrated a Robertsonian translocation between chromosomes 13 and 14 that accounted for his infertility. The psychopathological picture did not meet the criteria for a major depressive disorder but was characterized by symptoms of apathy or avolition. These symptoms were most probably aggravated by previous long lasting treatment with antidepressants.

Conclusions: Testosterone deficiency may be related to motivational deficits that should not be misunderstood for depressive illness. Apathy is probably an underestimated side effect of antidepressants.
} 


\section{Introduction}

In the sixties and seventies of the past century many studies have examined the chromosomal contribution to male infertility. Reported frequencies of chromosomal abnormalities range from $2.2 \%$ to $19.6 \%$ depending on the severity of male infertility ${ }^{1}$. Since the introduction of modern in vitro fertilization techniques, it is common practice to perform cytogenetic screening in patients with male infertility and chromosomal anomalies in up to $12 \%$ of cases are found of which the Robertsonian translocation 13;14 is the most frequent ${ }^{2-4}$. The majority of infertile males have normal levels of testosterone, $\mathrm{LH}$ and FSH. There is only one older case report about a slightly lower testosterone in a patient with this type of translocation ${ }^{5}$. In some infertile patients a primary testicular failure is present in which only low testosterone levels are found ${ }^{6}$.

It is well known that androgens like testosterone are implicated not only in sexual functions, but are thought to be also involved in aggression, social ranking and mood, although the data are difficult to interpret ${ }^{7,8}$. With respect to mood, it is hypothesized that testosterone modulates the activity of the hypothalamic pituitary adrenal axis ${ }^{9}$. Most studies about the relationship between mood and testosterone have been performed in aging men. It is still unclear whether there is a relationship between lower testosterone and major depression. Psychological dysfunctions such as irritability, fatigue, decreased sex drive, lack of motivation, and energy and a reduced feeling of well being are found that do not meet the criteria for a categorical diagnosis of major depression with or without melancholia $^{10-13}$.

In male patients with oligozoospermia and testosterone deficiency caused by a cytogenetic abnormality, hypogonadism is life long present and therefore the psychological consequences of low testosterone can be hypothesized to have a major impact on the development as a whole. Reports about hypogonadism with a genetic cause on development and psychological functioning are lacking.

In this case report, a male patient is described who had been treated for a depressive illness during several decades and in whom infertility due to a genetic abnormality and low testosterone was demonstrated. These findings are discussed in relation to the testosterone-depression debate.

\section{Case report}

The patient is a 57 year old male who was referred to the department of neuropsychiatry for re-evaluation of long lasting therapy resistant mood disturbances. He was born from non consanguine parents and had 3 younger sibs. His family history showed no mental disorders. During infancy and development there were no major somatic problems. His developmental history was characterized by a certain social withdrawal and lack of motivation in all domains. Although the patient completed both elementary and high school, his professional career showed a plethora of unfinished educations and non demanding jobs. From the age of 22 there were complaints about feelings of inferiority, mood instability and problems in task completion for which he visited several psychologists and psychiatrists. In subsequent years the patient had several short lasting relationships and sexual dysfunctions. He married aged 37 and he has a 12 years old son. At the age of 51 he was declared unable to work. Two years before, a diagnosis of bipolar 
affective disorder was made elsewhere for which he was treated with carbamazepine, Lithium and citalopram. During subsequent years treatment with various combinations of mood stabilizers and antidepressants did not improve his daily functioning and mood symptoms. The last 3 years his daily treatment consisted of $1500 \mathrm{mg}$ Lithium (0.9 $\mathrm{mmol} / \mathrm{l})$ and $1350 \mathrm{mg}(67 \mathrm{mg} / \mathrm{l})$ valproic acid.

Six years ago he developed obesity and insuline dependent diabetes mellitus type II, complicated by neuropathy that was confirmed with electromyography as well as an increased erectile dysfunction. Sildenafil was ineffective. Because of a low free testosterone (4.4 nmol/l; normal range: 10-28 $\mathrm{nmol} / \mathrm{l})$ and increased levels of LH (13,1 U/l; normal range: 2-9 U/1) and FSH (27,9 U/1; normal range:1,5-12 U/l), three years later a diagnosis of hypogonadism was made for which he was treated with testosterone once biweekly for 3 months without any effect on erectile function or general well being. For his diabetes mellitus the patient used insulin and oral antidiabetics.

At admission, the patient complained about fatigue, irritability, loss of libido, erectile dysfunction, problems in task completion and the inability to lead a self-serving life. He presented with a submissive and passive attitude, mental slowness and tiredness. Neuropsychiatric examination by means of a semi structured interview using the elements of the Comprehensive Psychiatric Rating Scale disclosed no major psychiatric disorder. Anamnestic information revealed infertility and his son was born after donor insemination. Extensive neuropsychological assessment demonstrated a total IQ of 128 (WAIS-III; verbal: 122; performal: 127), a mild to moderate impairment of attention/concentration with subsequent problems in the area of executive functioning. These mild cognitive dysfunctions were found to be embedded in a personality pattern characterized by self-devaluation combined with heightened sensitivity for critical evaluation, experiential avoidance, and low persistence. Somatic examination showed, apart from a BMI of 35 (length: $1.82 \mathrm{~m}$; weight: $115 \mathrm{~kg}$; waist circumference: $120 \mathrm{~cm}$ ), no abnormalities and normal genitals. Laboratory data showed slightly increased values for glucose and $\mathrm{HbA} 1 \mathrm{c}$ and a testosterone level at the lower margin of the reference values $(11,0$ nmol/l; normal range: 9-38 nmol/l). Because of his infertility, cytogenetic analysis was performed that demonstrated a Robertsonian translocation between the chromosomes 13 and 14 (figure 1) [karyotype: 45,XY, der $(13 ; 14)(\mathrm{q} 10 ; \mathrm{q} 10)]$.

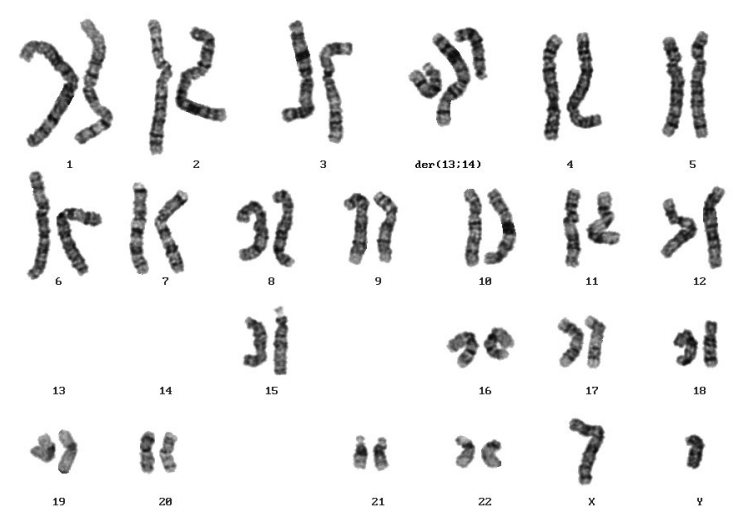

Figure 1. Robertsonian translocation 45,XYder(13;14)(q10;q10). 
During the clinical observation period, mood stabilizing agents were gradually tapered off and his medication for the diabetes mellitus was optimalized. At discharge the patient used insulin and oral antidiabetics and he reported an increased level of activity and feeling of well being.

Because of complete impotence, he underwent, after discharge, a second treatment with testosterone for 3 months that, however, was ineffective. At follow up after one year, the daily functioning of the patient was still characterized by lower psychological vitality, lack of motivation and irritability.

\section{Discussion}

In the here reported patient, referred for treatment refractory depression, cytogenetic analysis demonstrated a Robertsonian translocation between chromosomes 13 and 14 associated with infertility due to oligozoospermia. His medical history mentioned a testosterone deficiency, possibly related to the cytogenetic abnormality and aggravated by the diabetes mellitus type II. After discontinuation of all psychotropics, his daily functioning improved and returned to the level he had before the prescription of antidepressants and mood stabilizers. A second trial with testosterone did not improve his erectile dysfunction neither his psychological functioning. In retrospect, his obesity was aggravated by the use of antidepressants and mood stabilizers that resulted in insuline resistance and diabetes mellitus. Moreover, his avolition and apathy increased due to treatment with antidepressants and consequently he was declared unable to work at the age of 51. It cannot be ruled out that his low testosterone levels have caused reduced insulin sensitivity ${ }^{14}$.
Based on his history and the assessment of psychopathology and psychological functioning, a diagnosis of depressive disorder could not be established. In fact, his clinical condition could better be accounted for by the term 'apathy' or by the 'syndrome of avolition'. The syndrome of apathy comprises lack of motivation, diminished goal-directed cognition and reduced emotional concomitance of goaldirected behaviour ${ }^{15,16}$. It is often characterized by life long passivity, low role activity, low self esteem and low life satisfaction. Avolition is a psychological state characterized by severe problems in willed action, typically presenting with a general lack of desire, drive or motivation to strive for meaningful goals, and related to executive dysfunctioning. Symptoms of apathy or dysexecutive functioning can be induced or enhanced by antidepressants, especially SSRI's, and are reported in case series with children and adults ${ }^{17,18}$. In the epidemiological study by Van Geffen and coworkers, this phenomenon is listed as one of the 15 most frequently side effects reported by patients ${ }^{19}$.

Since the isolation of testosterone in the early thirties of the past century, this androgen has been associated with mood disturbances and subsequently used in the treatment of 'depression'20. During the past decades, several placebo-controlled clinical trials with testosterone have been performed in hypogonadal older men, demonstrating antidepressant like effects in some but not all studies ${ }^{21}$. It should be stressed, however, that these studies deal with mild depression and that the effects are only minimal. This can be illustrated by the study by Pope and coworkers who reported an antidepressant effect that was restricted to the non-affective symptoms of the Hamilton Depression Rating Scale but not present on the Beck Depression Inventory ${ }^{22}$. The effects of testosterone may therefore reflect the reversal of age related fatigue instead of a direct effect of this androgen on depression ${ }^{23}$. As stated by 
Amore, the relationship between testosterone deficiency and psychological dysfunctions may be the result of an interaction between biological and social changes and of the personal ability to adapt to age-related changes ${ }^{12}$.

In conclusion, symptoms of avolition and apathy are clinically relevant in hypogonadal men and should not be automatically placed in the expanding categorical concept of depression. Since such symptoms can be worsened by antidepressants, especially SSRI's, clinicians should be cautious in the prescription of these compounds, particularly in hypogonadal men.

\section{References}

1. Chandley AC. The chromosomal basis of human infertility. Br Med Bull 1979; 35: 181-186.

2. Mau UA, Bäckert IT, Kaiser P, et al. Chromosomal findings in 150 couples referred for genetic counselling prior to intracytoplasmic sperm injection. Hum Reprod 1997; 12: 930-937.

3. Tuerlings JHAM, De France HF, Hamers A, et al. Chromosome studies in 1792 males prior to intracytoplasmic sperm injection: the Dutch experience. Eur J Hum Genet 1998; 6: 194-200.

4. Peschka B, Leygraag J, Van der Ven K, et al. Type and frequency of chromosome aberrations in 781 couples undergoing intracytoplasmic sperm injection. Hum Reprod 1999; 9: 2257-2263.

5. Uccellatore F, Padova G, Squatrito S. Reproductive hormone studies in three subjects with a Robertsonian translocation. J Endocrinol Invest 1983; 6: 479-484.

6. Bhasin S. Approach to the patient. Approach to the infertile man. J Clin Endocrinol Metab 1995; 92: 1995-2004.

7. Rubinow DR, Schmidt PJ. Androgens, brain, and behavior. Am J Psychiatry 1996; 153: 974-984.

8. Mazur A, Booth A. Testosterone and dominance in men. Behav Brain Sci 1998; 21: 353-397.

9. Rubinow DR, Roca CA, Schmidt PJ et al. Testosterone suppression of CRH-stimulated cortisol in men. Neuropsychopharmacology 2005; 30: 1906-1912.

10. Delhez M, Hansenne M, Legros JJ. Andropause and psychopathology: minor symptoms rather than pathological ones. Psychoneuroendocrinology 2003; 28: 863-874.
11. Shores MM, Moceri VM, Sloan KL, et al. Low testosterone levels predict incident depressive illness in older men: effects of age and medical morbidity. J Clin Psychiatry 2005; 66: 7-14

12. Amore M. Partial androgen deficiency and neuropsychiatric symptoms in aging men. J Endrocrinol Invest 2005; 28: 49-54.

13. Tostain JL, Blanc F. Testosterone deficiency: a common, unrecognized syndrome. Nat Clin Pract Urol 2008; 5 : 388-396.

14. Kapoor D, Aldred H, Clark S, et al. Clinical and biochemical assessment of hypogonadism in men with type 2 diabetes. Diabetes Care 2007; 30: 911-917.

15. Marin RS. Differential diagnosis and classification of apathy. Am J Psychiatry 1990; 147: 22-30.

16. Marin RS. Apathy: a neuropsychiatric syndrome. J Neuropsychiatry Clin Neurosci 1991; 3: 243-253.

17. Hoehn-Saric R, Lipsey JR, McLeod DR. Apathy and indifference in patients on fluvoxamine and fluoxetine. $\mathrm{J}$ Clin Psychopharmacol 1990; 10: 343-345.

18. Garland EJ, Baerg EA. Amotivational syndrome associated with selective serotonin reuptake inhibitors in children and adolescents. Child Adolesc Psychopharmacol 2001; 11: 181-186.

19. Van Geffen ECG, Van der Wal SW, Van Hulten R, et al. Evaluation of patients' experiences with antidepressants reported by means of a medicine reporting system. Eur J Clin Pharmacol 2007; 63: 1193-1199.

20. Danziger L, Blank HR. Androgen therapy of agitated depressions in the male. Med Ann DC 1942; 1: 181-183.

21. Carnahan RM, Perry PJ. Depression in aging men. Drugs Aging 2004; 21: 361-376.

22. Pope HG, Cohane GH, Kanayama G, et al. Testosterone gel supplementation for men with refractory depression: a randomized, placebo-controlled trial. Am J Psychiatry 2003; 160: 105-111.

23. Schmidt PJ, Berlin KL, Danaceau MA, et al. The effects of pharmacologically induced hypogonadism on mood in healthy men. Arch Gen Psychiatry 2004; 61: 997-1004.

Address for correspondence:

Prof. Dr. W.M.A. Verhoeven

Vincent van Gogh Institute for Psychiatry

Stationsweg 46

5803AC Venray, The Netherlands

Phone: 0031.478527339

Fax: 0031.478527339

E-mail: wverhoeven@vvgi.nl 